UCRL-JC-127277

PREPRINT

\title{
Relativistic Self-Focusing in Underdense Plasma
}

\author{
M. D. Feit, J. C. Garrison, A. Komashko,
} S. L. Musher, A. M. Rubenchik, S. K. Turitsyn

This paper was prepared for submittal to the 13th Intemational Conference on Laser Interactions and

Related Plasma Phenomena

Monterey, California

April 13-18, 1997

April 24, 1997

This isa preprint of a paper intended for publication in a journal or proceedings. Since changes may be made before publication, this preprint is made available with the underatanding that it will not be cited or reproduced without the permiesion of the author. 


\section{DISCLAIMER}

This document was prepared as an account of work sponsored by an agency of the United States Government. Neither the United States Government nor the University of California nor any of their employees, makes any warranty, express or implied, or assumes any legal liability or responsibility for the accuracy, completeness, or usefulness of any information, apparatus, product, or process

disclosed, or represents that its use would not infringe privately owned rights. Reference herein to any specific commercial product, process, or service by trade name, trademark, manufacturer, or otherwise, does not necessarily constitute or imply its endorsement, recommendation, or favoring by the United States Government or the University of California. The views and opinions of authors expressed herein do not necessarily state or reflect those of the United States Government or the University of California, and shall not be used for advertising or product endorsement purposes. 
Relativistic Self-Focusing in Underdense Plasma

\author{
M.D.Feit 'J.C.Garrison' ${ }^{1}$,A.Komashko ${ }^{2}$, \\ S.L.Musher ${ }^{2}$,A.M.Rubenchik ${ }^{1}$, S.K.Turitsyn ${ }^{2}$ \\ 13th International Conference on Laser Interactions and Related \\ Plasma Phenomena \\ Monterey, CA \\ April 13-18, 1997.
}

1.Lawrence Livermore National Laboratory,P.O.Box 808, L-439, Livermore , CA 94550

2. Institute of Automation \& Electrometry, Novosibirsk, 630090, Russia

Work at LLNL was performed under the auspices of the U. S. Department of Energy by the Lawrence Livermore National Laboratory under Contract No. W-7405-Eng-48 


\title{
Relativistic Self-Focusing in Underdense Plasma
}

\author{
M.D.Feit ${ }^{1}$, J.C.Garrison ${ }^{1}$,A.Komashko ${ }^{2}$, \\ S.L.Musher ${ }^{2}$,A.M.Rubenchik ${ }^{1}$,S.K.Turitsyn ${ }^{2}$
}

1.Lawrence Livermore National Laboratory,P.O.Box 808, L-439, Livermore , CA 94550

2. Institute of Automation \& Electrometry, Novosibirsk, 630090 , Russia

\section{Introduction}

Recent advances in laser technology[1], based on the chirped pulse amplification technique, make intensities greater than $10^{18}$ $\mathrm{W} / \mathrm{cm}^{2}$ available for experiments. At such intensities, electron motion in the laser field is essentially relativistic and the physics of the laser-plasma interaction is very different from the well studied case of more moderate intensities.

In the present paper, we discuss light self-focusing in underdense $\left(n<n_{C}\right)$ plasmas. It was shown many years ago [2] that in the weakly relativistic regime, steady-state self-focusing is similar to self-focusing in a Kerr medium; the focusing is described by a Nonlinear Schrodinger Equation (NSE). For the beam power $P$ greater than a critical power $P_{c r}=16 n_{c} / n G W$, nonlinear self-focusing overcomes diffractive spreading and the beam is focused into a field singularity. More exactly, this behavior is followed up to the breaking of the paraxial approximation. Strong radiation scattering takes place after the focus.

If the initial beam power $P$ is well above $P_{c r}$, the laser beam first breaks into filaments with power $P$ about Pcr, each of which then undergoes catastrophic self-focusing.

Recent work [3-7], demonstrates that self-focusing changes qualitatively for very intense beams. In this case, the laser field becomes so strong that the ponderomotive force evacuates electrons from macroscopic regions (electron cavitation). Stable channeling can take place inside the resultant empty cavity since further focusing cannot take place. 
Understanding self-focusing is very important for realization of the "Fast ignitor " project.[8] In this ICF scheme, a powerful ultra short pulse produces a burst of energetic electrons to ignite an already compressed ICF target. Self-focusing and the subsequent anomalous scattering in the underdense plasma reduce fast electron generation efficiency. But radiation channeling can be very profitable for the "Fast ignitor" scheme. From the above arguments one can see that filamentation or channeling of radiation is determined not only by the beam power, but also by focusing conditions, density profile etc. The importance of propagation modeling for understanding is indicated by recent experiments $[9,10]$, in which similar experimental parameters resulted in radiation filamentation [9] or stable channeling [10].

Previous propagation studies must be advanced in two directions to be more reliable. First, as we will show, previously used descriptions of cavitation lead to non conservation of charge. Modification of the cavitation description and a comparison of our approach with previous results is presented in the first part of this paper. All previous studies treated the plasma ions as immovable. The exception was PIC simulations where simulation times were so short that ion dynamics was not important. We will show that ion motion is important even for picosecond pulse durations and a description of relativistic self-focusing including ion dynamics will be presented in second part of the paper. In particular, we will demonstrate the formation of empty, wide channels in underdense plasma in the wake of the laser pulse.

In the conclusion, we discuss the applicability of our results to real situations and possible consequences for the "Fast ignitor" project

\section{Cavitation and relativistic channeling}

Propagation of ultra-intense radiation in plasma involves a number of highly nonlinear phenomena characterized by different spatial and temporal scales e.g. the scale of charge separation, laser wavelength, transverse beam size, and longitudinal plasma scale. Also. the self-focusing of light in Kerr media is very different in 2D and 3D models so reliable modeling should be three dimensional.. As a result, even the best PIC simulation is limited, in practice, by short simulation times and small plasma volumes which don't match the experimental conditions [11]. On the other hand, the disparate sizes of parameters allows a simplified, average description [12,13]. Starting from the full coupled system of relativistic hydrodynamics 
for the electron and ion motions and Maxwell's equations, simplified equations for the envelope, averaged over rapidly varying phases, can be derived. . When the pulse duration is larger than $c / \omega_{p}$, further simplification which disregards charge separation in the direction of propagation is possible. Such a model does not include wake field generation, or Raman scattering-effects important for Laser acceleration studies. But for propagation in a plasma with density not very different from critical the approximation is good even for pulses with duration of a few tens of femtoseconds. We will show that in the process of self-focusing, small scale modulations of the pulse can arise. But the large variation in pulse durations $(0.5$ psec and up) used in modern experiments with powerful lasers allows reliable description even of pulse splitting.

in this approximation, the steady state relativistic self-focusing in the comoving system of reference is described by the paraxial equation $\{3-7]$

$$
2 i a_{z}+\Delta_{\perp} a+\left(1-\frac{n}{\gamma}\right) a=0
$$

Here $a$-is the envelope of the vector potential normalized as $a=$ $\mathrm{eA} / \mathrm{mc}^{2}, \gamma=\sqrt{1+\frac{|a|^{2}}{2}}$, transverse coordinates are normalized by $\mathrm{c} / \omega_{p}$ " and $z$ by $\frac{\omega c}{\omega_{p}^{2}} \gg \frac{c}{\omega}$. The electron density $\mathrm{n}$ in (1) is given by

$$
n=1+\Delta_{1} \gamma
$$

At high intensity, the ponderomotive force can overcome the electric field produced by charge separation in a macroscopic region and the density described by (2) drops down to zero and even can have nonphysical, negative values. To eliminate nonphysical negative density, it was suggested in ref[3] to set the electron density equal to zero inside the cavitation zone:

$$
\mathrm{n}=0 \text { if } 1+\Delta_{\perp} \gamma<0
$$

Such a model was used in the several investigations [3-7]. .Unfortunately, this model has a difficulty which makes quantitative results unreliable. To demonstrate the problem consider a powerful beam propagating in plasma, with ponderomotive forces evacuating electrons up to a radius $\mathrm{R}$. At $r=\mathrm{R}$ we must have $1+\Delta_{\perp} \gamma=0$. Together 
with the requirement of laser field decay at large $r$, and fixed beam power $\mathrm{P}, \mathrm{Eq}$ (1) with these condition completely determines the selfconsistent beam channeling[6]. Now consider the condition of charge conservation

$$
\int(n-1) d V=0
$$

This condition makes our equation over determined and solutions with cavitation obtained in [3-6] have, generally speaking, non-zero charge. In Fig.(1), we plot the net charge of the steadystate solution of $\mathrm{Eq}(1)$ vs the power of the channeling beam. We see that charge non conservation starts just after the appearance of and increases with increasing channeling power. The total charge is negative.

The above inconsistency will be removed by the introduction of surface charge on the cavitation boundary which makes Eq (1) consistent with both the boundary conditions and the additional condition (4).. A more natural way to solve the problem is to take into account the finite plasma temperature. In this case, instead of $\mathrm{Eq}(2)$ one can derive the equation

$$
n=1+\Delta_{\perp}(\gamma+\alpha \ln n) ; \alpha=\frac{T}{m c^{2}} \ll<1
$$

Eq (5) has a simple physical meaning. The displacement of electron density produces the electrostatic potential $\Phi$, according to the relation $\Delta_{1} \Phi=1-n$. Hence, Eq (5) describes a Boltzmanian electron distribution in joint electrostatic and ponderomotive potential. It is clear that in this case the electron density in the region of laser field localization can be extremely small, but nevertheless nonzero and we will have no singularities in the solution.

Figs.(2) and (3) compare the evolution of initially Gaussian beams propagated in plasma as described by the cavitation model and by the system Eqs(1) and (5). Fig.(2) compares the laser field amplitude, and Fig.(3) the electron density distribution. One can see that while being qualitatively similar, the solutions are very different quantitatively. Our charge conserving solution is smoother, and the channel is wider.. One sees that the regular solution does not exhibit the high spikes of laser intensity typical of the cavitation model simulation [5].

Eqs (1), (5) can be written in Hamiltonian form 


$$
2 i a_{z}=\frac{\delta H}{\delta a^{*}}
$$

with the Hamiltonian

$$
\mathrm{H}=\int\left[\left|\nabla_{1} a\right|^{2}-|a|^{2}+2(m-1)-(n-1) \Delta_{1}^{-1}(n-1)+2 \alpha(n \ln n-n+1)\right] d V
$$

with additional constraint $\frac{\delta H}{\delta n}=0$, equivalent to Eq. (5). The Hamiltonian structure means that besides the power $P=\int|a|^{2} d V$, Eqs (1), (5) also conserve a Hamiltonian $\mathrm{Eq}(7)$. Here, we have another difference from the cavitation model. Outside the cavitation zone, the equations still have Hamiltonian structure. $\mathrm{H}$ is given by (7) with $\alpha=0$.

The equations are also Hamiltonian inside the cavity, but due to the surface effect, the Hamiltonian isn't conserved within the cavitation model. The evolution of theHamiltonian is presented in Fig. (4). The jumps in $\mathrm{H}$ values are directly correlated with cavitation appearence.

Hamiltonian (7) was conserved with high accuracy during the evaluation of (1),(5)

Consider steady state solutions of Eq(1). Similar to steady state solutions for the NSE, they realize extremums of $\mathrm{H}$ for fixed values of P. (see e.g. the review [11]). It was shown in [15] that for $\alpha=0, \mathrm{H}$ is bounded from below for fixed values of $\mathrm{P}$. Small thermal corrections don't change this result. The boundedness of $\mathrm{H}$ means that the solution corresponding to minimum $\mathrm{H}$ for a fixed $\mathrm{P}$ is stable, according to the Lyapunov theorem[14].

It appears, at first, impossible to arrive at this stable solution from general initial conditions. The initial value of the Hamiltonian is different from the $\mathrm{H}$ value for the steady state solution and $\mathrm{H}$ is a conserved quantity. However, this difference can be removed by radiation out of the channel. It means the efficiency of trapping in the channeling regime can be sensitive to focusing conditions,plasma density etc.

We demonstrated that an adequate description of cavitation doesn't qualitatively change the results of previous studies. Stable channeling of intense laser radiation through underdense plasma is possible if the laser beam is powerfull enough and tightly focused. But channel width, axial intensity, and distribution smoothness differ quantitativly from previous publications. 


\section{Relativistic propagation, including ion dynamics}

It seems natural to disregard ion motion in studies of ultrashort pulse propagation. But we show below that ion motion becomes essential even for pulses of picosecond duration. Two effects are important. First, self-focusing creates very narrow channels so even small ion displacement effects light propagation. The ponderomotive pressure is so strong that ions acquire high velocity during the pulse. Also, ions kicked out during the pulse, continue to move after the pulse termination. The resulting empty channel in the plasma is important for the "Fast Ignitor" project. The later effect means that the assumption of steady state propagation must be given up to include ion dynamics. In comparison with the previous studies we must describe a 3D problem, with $z, t$ and $r$ variables. But because we describe longer pulses, the ions have time to follow the electrons and the charge separation effects discussed above are not important. Instead of Eqs(1), (2) the following set of equations must be solved

$$
\begin{aligned}
& {\left[\nabla_{\perp}^{2}+2 i k \partial_{\zeta}\right] a_{L}=\left(n_{e}+\frac{1}{M} n_{i}\right) a_{L}} \\
& \partial_{\tau} u+\frac{1}{2} \nabla_{\perp} \mathbf{u}^{2}=-\frac{1}{M} \nabla_{\perp} \gamma \\
& \partial_{\tau} n_{i}+\nabla_{\perp} \cdot\left(n_{i} u\right)=0 \\
& n_{e}=\max \left[\frac{n_{i}+\nabla_{\perp}^{2} \gamma}{\gamma}, 0\right]
\end{aligned}
$$

where $\mathbf{k}$ is the laser wavenumber in plasma units, $M=m_{i} /\left(m Z_{i}\right)$, and $u$ is the ion velocity in units of $c$. The neglect of thermal effects can be justified by the observation that the preformed plasma is expected to have a temperature of at most a few $\mathrm{keV}$, and collision times long compared to the pulse duration. Thus the thermal pressure will be negligible compared to the ponderomotive pressure, and the laser energy will be deposited in the plasma in the form of fluid motion rather than heating.

Before discussing the simulation results, it is instructive to make some rough estimates. In conventional units the transverse ion acceleration is $\alpha_{\perp}=-\left.\mathrm{mc}^{2} \nabla_{\perp} \mathrm{al}_{L}\right|^{2} /(4 \mathrm{M} \gamma)$, so $\alpha_{\perp} \approx \mathrm{mc}^{2}\left|\mathrm{a}_{\mathrm{L}}\right|^{2} /(4 \mathrm{M} \gamma \mathrm{w})$, where $w$ is the beam radius. Consequently the transverse velocity after a time $t$ is approximately $v(\tau)=m c^{2} \tau\left|a_{L}\right|^{2} /(4 M \gamma w)$ The time required for expulsion of the ions $\left(\tau_{\text {cav }}\right)$ is defined by $v\left(\tau_{\text {cav }}\right) \tau_{\text {cav }}=w$ so c $\tau_{\text {cav }}=2 \sqrt{\mathrm{M} \gamma / \mathrm{m}} \mathrm{w} /\left|\mathrm{a}_{\mathrm{L}}\right|$, and the velocity at this time is $v_{\text {cav }}=c\left|a_{L}\right| /(2 \sqrt{\mathrm{M} \gamma / \mathrm{m}})$. For the intensity range

$10^{19} \mathrm{~W} / \mathrm{cm}^{2} \geq \mathrm{I} \geq 10^{17} \mathrm{~W} / \mathrm{cm}^{2}$, one finds $2 \mathrm{ps} \leq \tau_{\mathrm{cav}} \leq 20 \mathrm{ps}$ and 
$10^{-2}>v_{\text {cav }} / \mathrm{c}>10^{-3}$. The cavity will continue to expand for some time after the pulse has passed. We estimate the final cavity radius by equating the energy deposited by the pulse to the PdV work done in creating a cavity of radius $R$ in a fluid with temperature $T$, with the result $. R / w=\sqrt{\mathrm{mc}^{2} /\left(8 \mathrm{k}_{B} \mathrm{~T}\right)}\left|\mathrm{a}_{L}\right|$ Then $a=O(1)$ yields $R>>w$, i.e., the cavity radius will be larger than the spot size of the beam. After the pulse has passed, the cavity will collapse in the time $T_{c l p}=R / v$ th , where $v_{t h}=\sqrt{k_{B} T / M}$. For the values considered below,.$T_{\text {clps }} \approx 10^{2}$ ps $B y$ contrast, the electrons-only cavity, which requires a charge separation between electrons and ions, will collapse as the pulse is passing. The energy stored in the electrons is thereby returned to the field which experiences no loss during propagation. In the case of complete cavitation, the rate of energy loss from the pulse is estimated by equating the difference in intensity at two planes, separated by $\Delta z$, to the rate of energy deposition in the cylindrical volume defined by the planes. This yields $\mathrm{dI}_{\mathrm{L}} / \mathrm{dz}=-\mathrm{n}_{\mathrm{i}}\left(\mathrm{M} \mathrm{v} \mathrm{v}_{\mathrm{cav}}^{2} / 2\right) / \tau_{\text {esc }}$, where the escape time is $\tau_{\text {esc }}=w / v_{c a v}$. In terms of $\left|a_{L}\right|$ this equation becomes $\left|\mathrm{a}_{\mathrm{L}}\right|^{-2} \mathrm{~d}\left|\mathrm{a}_{\mathrm{L}}\right|^{2} / \mathrm{dz}=-\left(1 / \mathrm{L}_{0}\right)\left(\left|\mathrm{a}_{\mathrm{L}}\right| / \gamma^{3 / 2}\right)$, where $L_{0}=8 \frac{n_{C r}}{n_{i}} \sqrt{\frac{M}{m}} w$, and $\mathrm{n}_{\mathrm{cr}}$ is the critical density for the laser frequency. Thus the absorption length is $L_{a b s}=\gamma^{3 / 2} L_{0} /\left|a_{L}\right|$. We compare $L_{a b s}$ to $L_{R}$, the characteristic diffraction length for transverse modulations on the scale of the plasma wavelength, which is defined by $L_{R}=k_{k} p^{-2}=n_{C r} / n_{k}$. The result is $L_{a b s} / L_{R}=8 \sqrt{M / m} k_{P} w \gamma^{3 / 2} /\left|a_{L}\right| \geq 9.12 \sqrt{M / m} k_{P} w ~ \gg 1$, i.e., the pulse can propagate for many diffraction lengths before suffering significant loss.

The propagation equation is solved by a spectral method for paraxial propagation [16]. The momentum-balance equation is approximated by a finite-difference scheme using upwind differencing, and the continuity equation (is treated by integrating it over concentric spherical shells surrounding the radial grid points, with fluxes defined by the same upwind scheme. In the results presented below we impose cylindrical symmetry and use the incident laser field $a_{L}=a_{L 0} \exp \left[-r^{2} /\left(2 w^{2}\right)\right] \exp \left[-\tau^{2} /\left(2 T_{p}^{2}\right)\right]$ The laser wavelength, spot size and pulse duration are respectively, $\lambda=1.06 \mu \mathrm{m} . w=7 \mu \mathrm{m}$ and $\mathrm{T}_{\mathrm{p}}=60 \mathrm{psec}$ in all cases.

For the lowest intensity, $I=10^{17} \mathrm{~W} / \mathrm{cm}^{2}$, strong self focusing effects can be seen in Fig. 5., which shows the temporal profile of the laser intensity on axis for three values of the propagation depth. The temporal modulation, which is similar to results obtained in calculations for Kerr media [17], is a consequence of the variation in 
self focusing lengths during the pulse. There are also important differences from the case of Kerr media; in particular the formation of the channel prevents truly singular self focusing. The severe pulse distortion shown in Fig. 5. does not prevent the formation of a cavity. This is seen in Fig.6 which shows the ion density as a function of radius, $r$, at various times, $\tau=t-z / c$, for the same propagation depths. The time required for the formation of the cavity and the cavity radius agree with the estimates, and the shock front forming the wall of the cavity at each time is clearly evident. The plots in Fig. 6. show that the increased intensity due to self focusing causes more rapid cavity formation as the pulse propagates, but the cavity radius at a given time $t$ may be decrease somewhat during propagation, as seen by comparison of the plots for $z=30$ and $60 \mathrm{~mm}$.

At the highest intensity, $I=10^{19} \mathrm{~W} / \mathrm{cm}^{2}$, the destructive effects of self focusing are largely suppressed, as seen in Fig. 7.. The temporal modulation of the pulse increases with propagation depth, but it is now confined to the leading edge where the intensity is low. In this case self focusing is self limiting, since the increase in the intensity at the leading edge drives rapid cavity formation so that the following part of the pulse effectively propagates in a vacuum and experiences no self focusing. This is an improvement over the low intensity behavior, but the pulse shape will eventually be degraded at any intensity. The fraction of the pulse experiencing modulation increases by roughly $2 \%$ for each $10 \mathrm{~mm}$ of propagation length, so even the high intensity pulse will be severely deformed after propagating several hundred microns. Cavity formation is similar to the low intensity case, but occurs more quickly, in line with the estimates.

These simulations show that intense laser pulses propagating in an underdense plasma will produce completely evacuated and longlived channels. The longitudinal profile of the pulse is distorted by self focusing, but at higher intensities the rapid formation of the cavity tends to suppress this effect. Our results support a fast ignitor concept in which an intense pulse of a few ps duration creates an evacuated channel which will persist for time about 100 psec.. The heating pulse can then propagate in the channel without loss or distortion. In a subsequent publication we will present a more detailed account including applications to non-Gaussian beam shapes.

Discussion 
Pulses with power much greater than the critical power for self focusing $\mathrm{P}_{\mathrm{cr}}$ can form stable channels through underdense plasma. But the beam power is not the only parameter characterizing propagation. A defocused or weakly focused beam can undergo filamentation during propagation through very underdense plasma.

If the pulse duration exceeds a few picoseconds, total plasma evacuation and creation of a wide empty channel, suitable for transportation of the short heating pulse in the "Fast Ignitor" scheme takes place. If the pulse is long and intense, most of the pulse is transported to the critical surface and can be used to bore into the overdense plasma. Further optimization of the energy distribution between the channeling and heated pulses can be done with consideration of the processes in overdense plasma

Work at LLNL was performed under the auspices of the U. S. Department of Energy by the Lawrence Livermore National Laboratory under Contract No. W-7405-Eng-48

References

1. M.Pery, G.Mourau Science, 64, 1917, (1994)

2. A.Litvak Sov.Phys JETP,30,344,(1969) , A.Berhoer, V.Zakharov Sov.Phys JETP 31.486.(1970), C.Max, J.Arons, A.B.Langdon Phys.Rev.Lett 33,209,(1974)

3.G.-Z. Sun, E. Ott, Y. C. Lee, et al., Phys. Fluids 30, 526 (1987).

4. X.L.Chen, R.N.Sudan Phys Fluids B 5.1336,(1993)

5.A. B. Borisov, A. V. Borovskiy, O. B. Shiryaev, et al., Phys. Rev. A 45, 5830 (1992)., A. B. Borisov, O. B. Shiryaev, A. McPherson, et al., Plasma Phys. Control. Fusion 37, 569 (1995).

6.A. Komashko, S. Musher, S. Turitsyn, et al., JETP lett. 62,860 (1995).

7.P. Sprangle, E. Esarey, J. Krall, et al., Phys. Rev. Lett. 69, 2200 (1992).

8.M. Tabak, J. Hammer, M. Glinsky, et al., Phys. Plasmas 1, 1626 (1994)

9 P.E.Young, R.P.Bolton Phys.Rev.Lett. 77.4556.1996

10. M.Borghesi, A.J.MacKinnon et al, Phys.Rev.Lett 78.879.1997

11. A. Pukhov and J. Meyer-ter-Vehn, Phys. Rev. Lett. 76, 3975 (1996)

12. E.Esarey,P.Sprangle, J.Krall, A.Ting IEEE Transaction on Plasma Science, 24.252 (1996)

13.M. D. Feit, J. C. Garrison, and A. M. Rubenchik, Phys. Rev. E 53, 1068 (1996). 
14. Kuznetsov E.A.,Rubenchik A.M., Zakharov V.E. Phys.Reports $142,105,(1986)$

15. X.L.Chen, R.N.Sudan Phys.Rev.Lett. 70.2082.(1993)

16. M. D. Feit and J. A. Fleck, Opt. Lett. 14, 662 (1989).

17. M. D. Feit and J. A. Fleck Jr., J. Opt. Soc. Am. B 5, 633 (1988).

\section{Figure Captions}

Figure 1: Total charge $Q=\int(1-n) d V$ of steady-state solution (1) with standard cavitation description (3) vs trapped power. Charge non conservation starts just after cavitation appearance and grow up with increase of channeling power. Total charge is negative. Dotted line -cavitation radius

Figure 2: Evolution of the amplitude $a(r, z)$ of the vector potential for the pulse with power $\mathrm{P} / \mathrm{Pcr}=118.5$ and the initial distribution $\mathrm{a}(\mathrm{z}=0, \mathrm{r})=12 \exp \left[-(\mathrm{r} / 3.5)^{2}\right]$ a-cavitation model,b-regular description (5)

Figure 3: Electron density evolution for the same case.

Figure 4: Hamiltonian evolution. The jumps of Hamiltonian coinsides with cavitation appearence

Figure. 5: Normalized laser intensity on axis, vs.t , at $z=30,60$, and $90 \mathrm{~mm}$, for an incident pulse with $\mathrm{I}=10^{17} \mathrm{~W} / \mathrm{cm}^{2}$ and a temporal profile shown by the dashed curve. The normalization intensity, corresponds to $a_{1}=1$

Figure 6:. Snapshots of the normalized ion density, , vs. r (mm) at several values of $t$ for $z=0,30,60$, and $90 \mathrm{~mm}$, for the same case as Fig. 5. The horizontal dashed line represents the initial density, and the number next to each curve gives the snapshot time in ps.

Figure 7 Normalized laser intensity on axis, vs. $t$, for an incident pulse with $\mathrm{I}=10^{19} \mathrm{~W} / \mathrm{cm}^{2}$. Other parameters as in Fig. 5. 


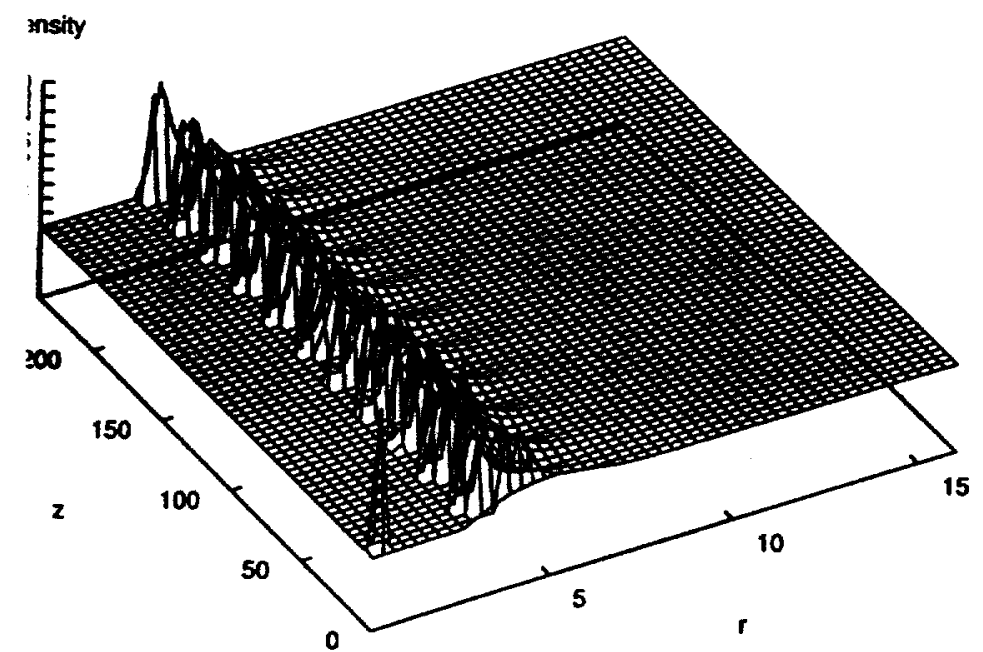

a

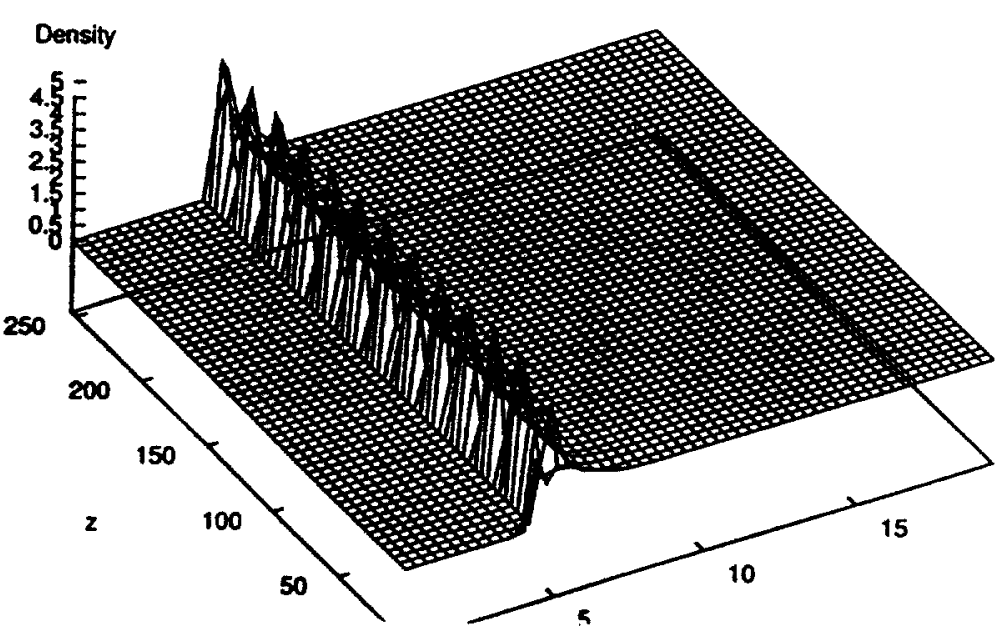

b

Fig.3

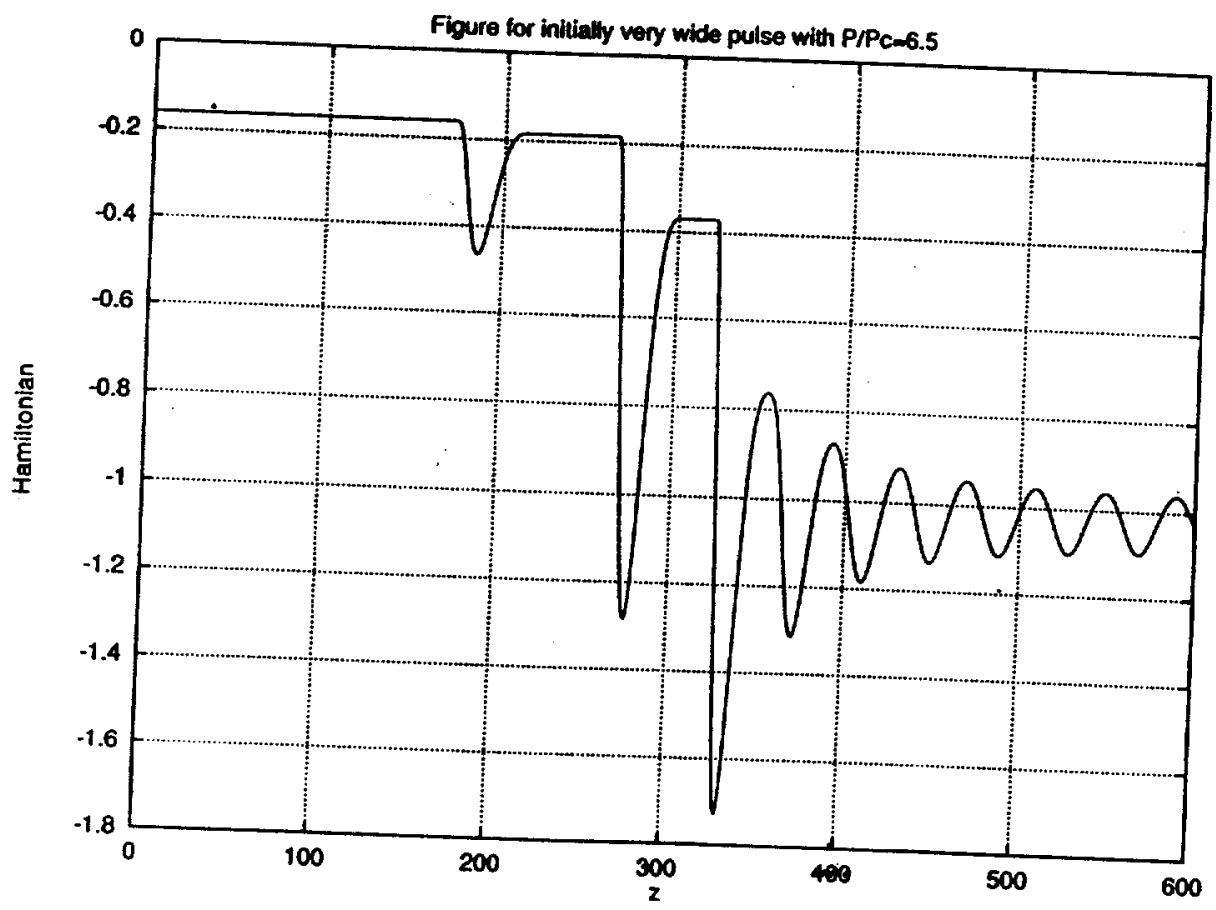

Fig.4 

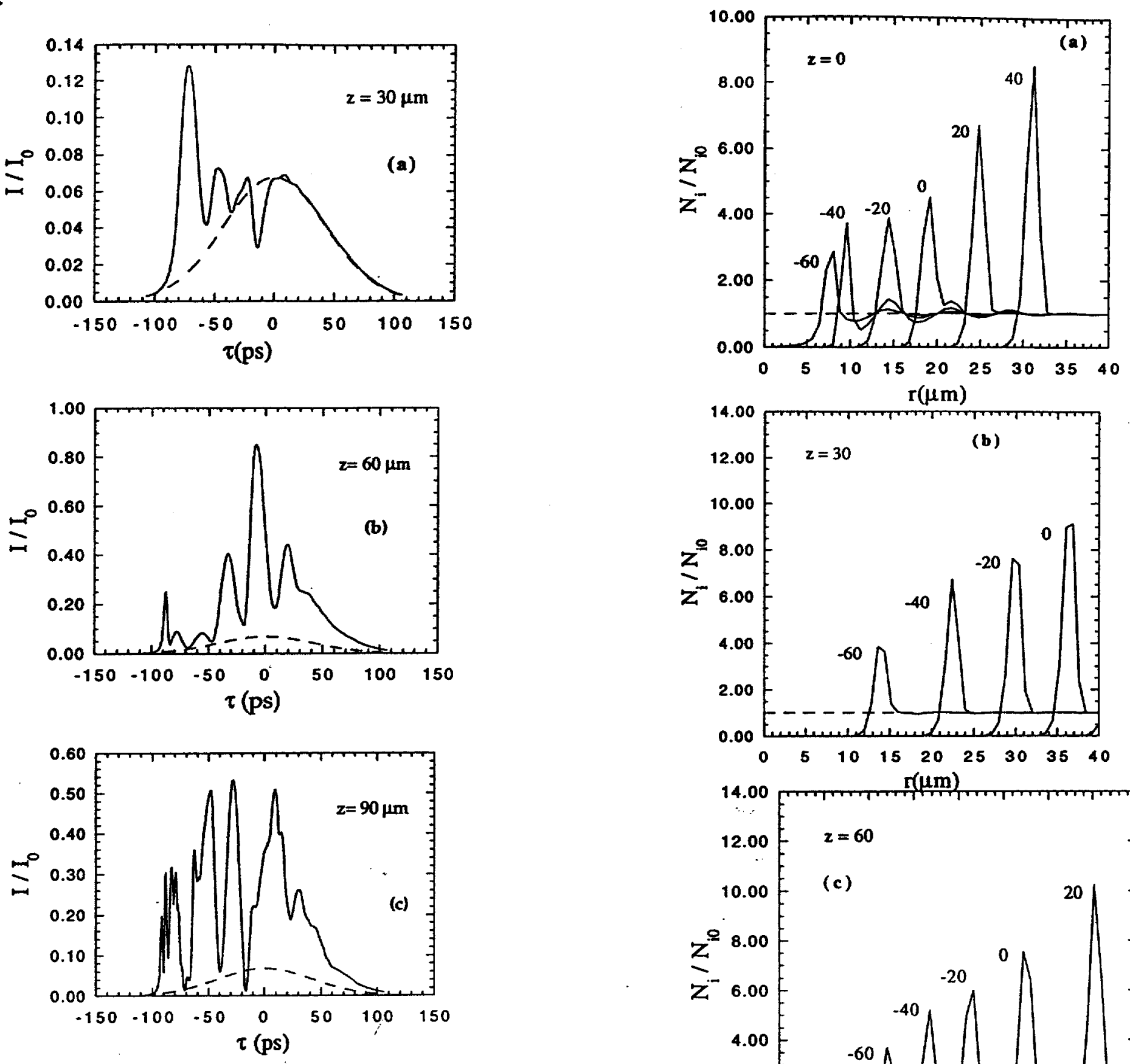

Fig.5
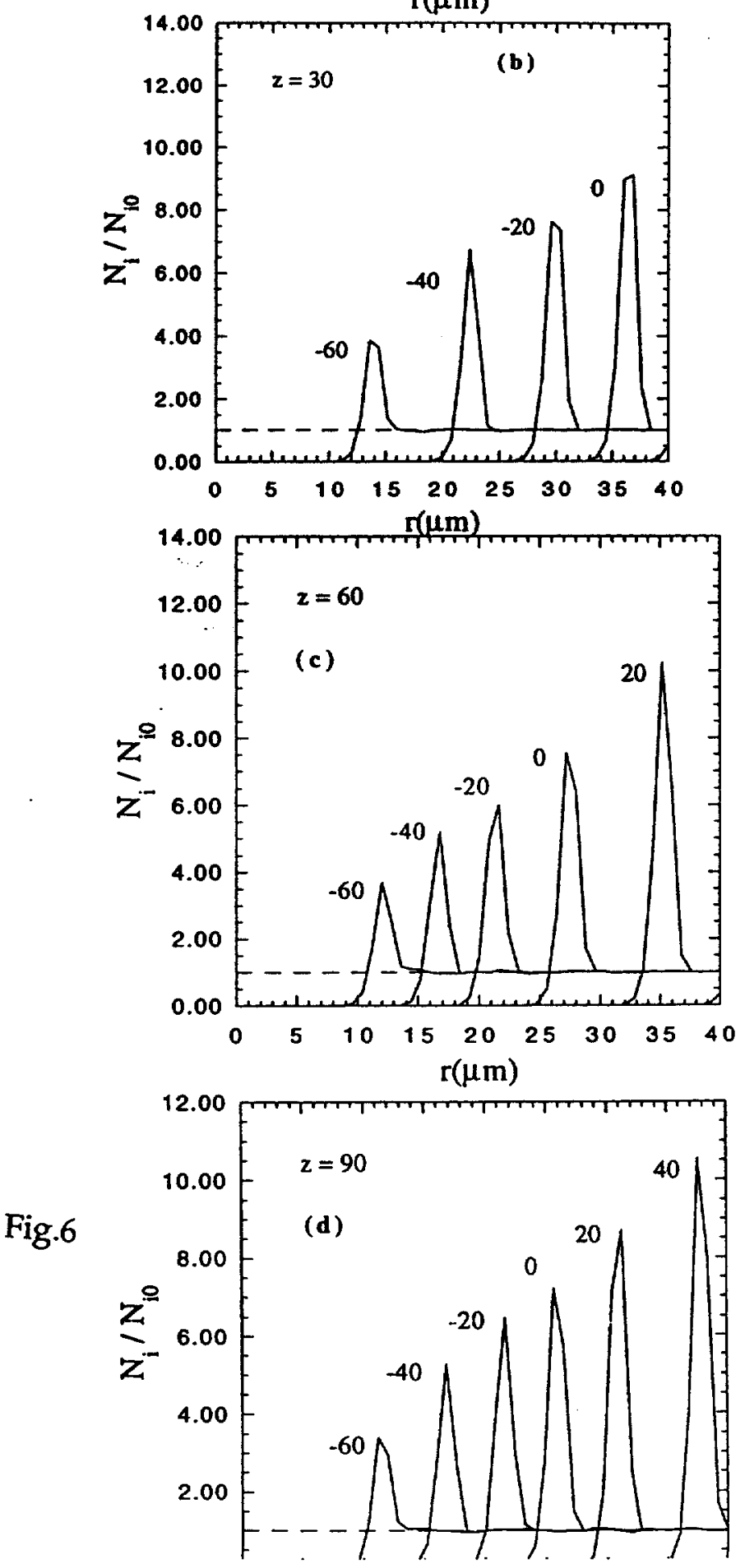

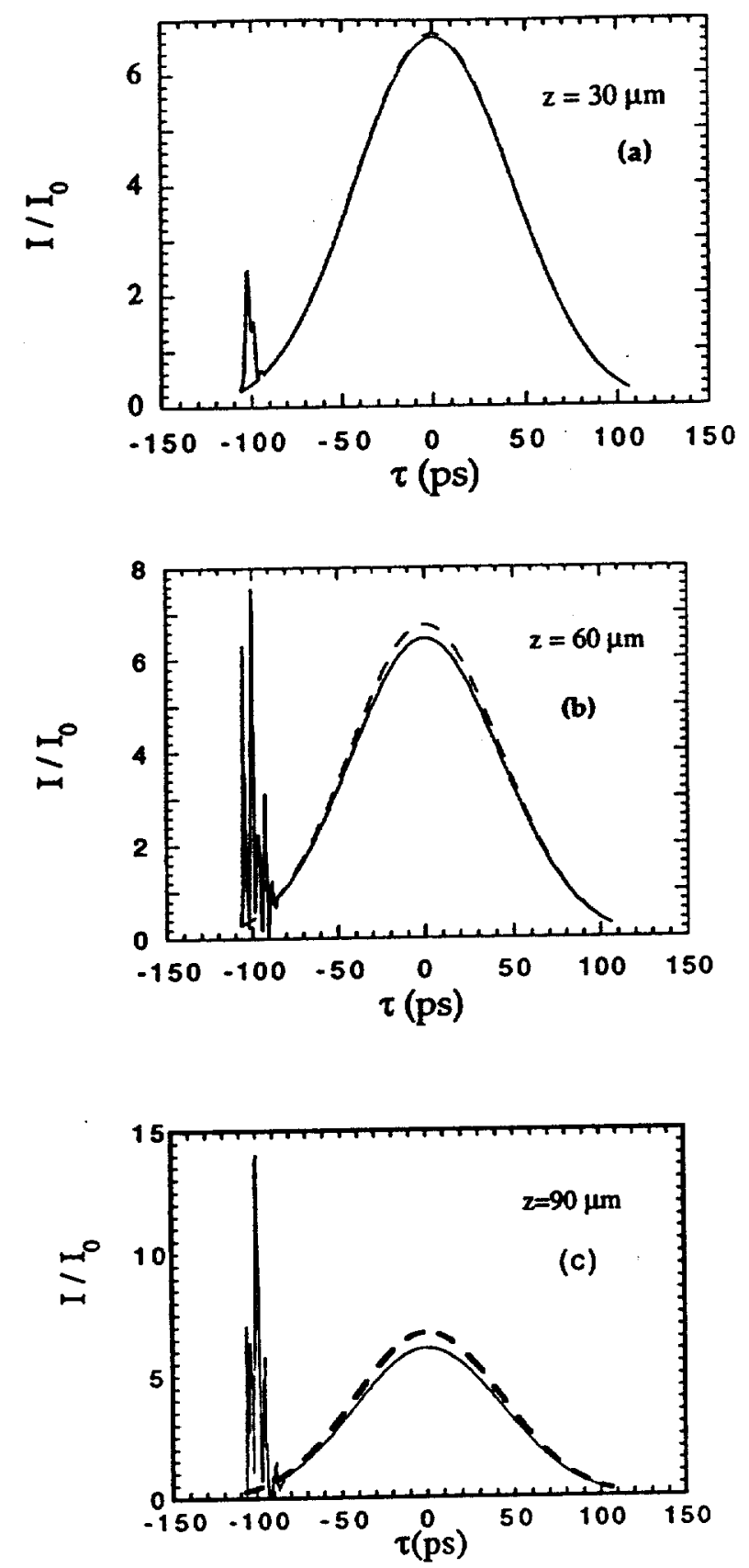

Fig.7 


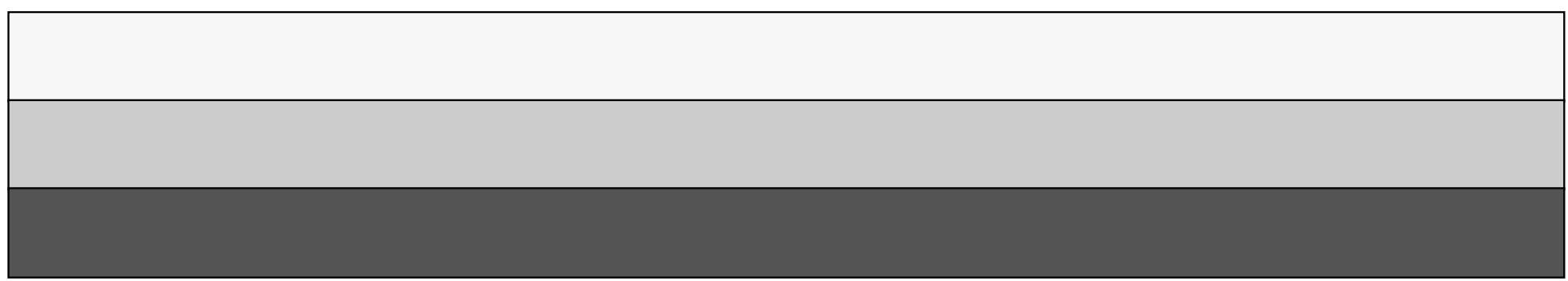

